\title{
Cementos Óseos Acrílicos Modificados con Hidroxiapatita/ Acetato de Vinilo. Caracterización Mecánica, Termoanálitica y Bioactividad In Vitro
}

\author{
Nayrim B. Guerra, Mónica L. Hernández, Ramón G. Santos \\ Laboratorio de Biomateriales, Centro Nacional de Investigaciones Científicas
}

\begin{abstract}
Resumen: Los cementos óseos se han convertido en los últimos años en biomateriales de gran utilidad en la fijación de prótesis y en la reconstrucción del hueso. El objetivo de este trabajo es evaluar las propiedades termoanalíticas tales como temperatura máxima de polimerización y tiempo de fraguado en cementos óseos acrílicos modificados con hidroxiapatita/ acetato de vinilo, determinar la resistencia a la compresión axial y realizar ensayos de bioactividad in vitro. Diferentes contenidos de acetato de vinilo fueron incorporados en cementos óseos acrílicos cargados todos con un $30 \%$ de hidroxiapatita CORALINA ${ }^{\circledR} \mathrm{HAP}-200$. Las propiedades mecánicas y los parámetros de curado fueron evaluados cumpliendo lo establecido en la Norma ISO 5833 descrita para cementos óseos acrílicos. Se determinaron los parámetros termoanalíticos, obteniéndose tiempos de fraguados entre 3 y 6 minutos y los valores de temperaturas máximas de polimerización oscilan entre 66 y $88^{\circ} \mathrm{C}$. Se obtuvo formulaciones con valores de resistencia a la compresión superiores a lo establecido en la Norma ISO 5833. Se demostró la bioactividad de las formulaciones mediante la inmersión de las muestras en fluido biológico simulado, observándose en la superficie de las mismas la nucleación y el crecimiento de cristales con morfología similar a las apatitas biológicas.
\end{abstract}

Palabras-clave: Cementos óseos acrílicos, hidroxiapatita, tiempo de fraguado, resistencia a la compresión.

\section{Acrylic Bone Cement Modified whit Hydroxiapatyte/Vinyl Acetate. Mechanical, Thermoanalytical Characterization and In Vitro Bioactivity}

\begin{abstract}
Bone cements have become biomaterials of great utility in the prosthesis fixation and as substitutes to the bone. The objective of this study is to evaluate the thermo analytical properties such as setting time and peak temperature of polymerization of acrylic bone cement modified with hydroxyapatite/vinyl acetate, to determine the compression strengths and perform in vitro bioactivity tests. Amounts of vinyl acetate component were incorporated in different percentages in acrylic bone cements, all loaded with $30 \%$ of hydroxyapatite CORALINA ${ }^{\circledR}$ HAP-200. Curing parameters and mechanical properties were determined finding formulations that comply with the ISO 5833 norm. In vitro bioactivity tests were performed in two cement formulations. The setting time obtained ranged between 3 and 6 minutes and the peak temperature varied between 66 and $88^{\circ} \mathrm{C}$. Compression strengths were higher than established in the ISO 5833 regulation for mechanical properties evaluation of acrylic bone cements. The bioactivity was demonstrated by immersion of the samples in simulated body fluid, in which nucleation and crystal growth were observed on the sample surface with similar morphology and composition to biological apatite.
\end{abstract}

Keywords: Acrylic bone cement, hydroxyapatite, setting time, compression strengths.

\section{Introducción}

Los cementos acrílicos autopolimerizables se han usado ampliamente en odontología y cirugía ortopédica como fijadores de prótesis, como agentes de relleno de cavidades óseas, como separadores en cirugía de reemplazo e incluso se han propuesto para otros usos como dispositivos médicos en el tracto urinario. ${ }^{[1-3]}$

Estos cementos tradicionalmente se presentan como un conjunto formado por una parte sólida, usualmente en forma de perlas de poli (metacrilato de metilo), peróxido de benzoilo (iniciador de la reacción) y sulfato de bario u óxido de zirconio (como agente radiopaco) y una parte líquida constituida por el monómero metacrilato de metilo y la $N, N$-dimetil-p-toluidina (activador de la reacción de polimerización). Aunque estos cementos aun se utilizan intensamente, se reportan varias desventajas asociadas a los mismos. ${ }^{[4]}$ Entre ellas se encuentra la necrosis térmica del hueso, necrosis química debido al monómero liberado que no ha reaccionado en la polimerización, contracción del cemento durante la polimerización, mala distribución del cemento alrededor del implante y escasa adhesión en

Autor para correspondência: Nayrim B. Guerra, Laboratorio de Biomateriales, Centro Nacional de Investigaciones Científicas, Avenida 25 y Calle 158, Playa, Apartados Postales 6412 - 6414, Ciudad de La Habana, Cuba. E-mail: nayrim.brizuela@cnic.edu.cu 
las interfase hueso-cemento, cemento-prótesis, entre otros. A pesar de estas dificultades prácticas, dichos materiales siguen siendo utilizados en la práctica médica debido a las excelentes propiedades mecánicas que presentan.

La determinación de las propiedades mecánicas en este tipo de material constituye un factor muy importante a tener en cuenta. Según las condiciones de ensayo, geometría de la probeta y modo de solicitación mecánica, se pueden diferenciar dos tipos de ensayos: estáticos y dinámicos. ${ }^{[5]}$ En este trabajo se realizaron ensayos estáticos; específicamente la determinación de la resistencia a la compresión axial. Para evaluar los resultados experimentales se utilizó como referencia la Norma ISO 5833 establecida para cementos óseos acrílicos. ${ }^{[6]}$

De las propiedades termoanalíticas, las fundamentales son el tiempo de fraguado, por la importancia que tiene en el acto quirúrgico y la temperatura máxima de polimerización, por los efectos secundarios que podría ocasionarle al paciente. El tiempo de fraguado de los cementos óseos según la ASTM F541-99 ${ }^{[7]}$ debe estar comprendido entre los 5 y 15 minutos y entre 3 y 15 considerando la Norma ISO 5833 ${ }^{[6]}$ (Tabla 1). La reacción de polimerización que ocurre en los cementos óseos durante el proceso de fraguado es exotérmica y el calor desprendido es de 54,4 $\mathrm{kJ} . \mathrm{mol}^{-1}{ }^{[8]}$ Se conoce que los altos calores de polimerización pueden causar necrosis del tejido en los alrededores de la prótesis y además puede afectar severamente la durabilidad del implante debido a que induce la reabsorción del tejido óseo y produce aflojamiento de la prótesis. ${ }^{[9]}$

Elestudio de labioactividad in vitro es un ensayoimportante a valorar en este tipo de biomateriales. ${ }^{[8]}$ Su determinación se fundamentan en el hecho de que en los materiales cerámicos que se enlazan directamente al hueso se ha podido demostrar que las primeras etapas del mecanismo de enlace pueden simularse en un medio inorgánico, sin presencia de células, siempre y cuando dicho medio contenga los iones necesarios para que el material pueda desarrollar una nueva fase tipo carbonato-hidroxiapatita (CHA) en su superficie.

En este trabajo se estudió el efecto de la incorporación de acetato de vinilo en cementos óseos acrílicos rellenos con un $30 \%$ de hidroxiapatita CORALINA ${ }^{\circledR}$ HAP - 200 sobre la bioactividad in vitro, la resistencia a la compresión axial y las propiedades de curado (tiempo de fraguado y temperatura máxima de polimerización).

\section{Materiales y Métodos}

\section{Preparación de cementos óseos acrílicos}

Los cementos se prepararon mediante una polimerización radicálica a partir de una fase sólida y una líquida. Como parte sólida se utilizó poli (metacrilato de metilo) (PMMA) [FERSOdental, Cuba] el cual contiene 1,2\% de peróxido de benzoilo (PBO) como iniciador de la reacción de polimerización. Como relleno inorgánico se empleó la Hidroxiapatita CORALINA ${ }^{\circledR}$ HAP-200 $^{[10]}$ con un tamaño de partícula entre 56 y $80 \mu \mathrm{m}$, suministrada por el Departamento de Producción de Biomateriales del Centro Nacional de Investigaciones Científicas.

La parte líquida está constituida por metacrilato de metilo (MMA) procedente de la firma [FERSOdental, Cuba] la cual contiene $0,4 \%$ de $N, N$-dimetil- $p$-toluidina (DMpT) como activador de la reacción de polimerización. Además se adicionaron diferentes proporciones del monómero acetato de vinilo (AcV) [Merck], el cual fue previamente purificado por destilación a presión atmosférica, a una temperatura de $72,5^{\circ} \mathrm{C}$.

Se prepararon nueve formulaciones diferentes aplicando un diseño de experimento $3^{2}$ (Tabla 2), donde las variables estudiadas fueron: contenido de acetato de vinilo (A) y relación sólido/líquido empleada en la preparación las formulaciones (B).

\section{Caracterización mecánica}

La resistencia a la compresión se determinó en una máquina universal de ensayos Material Test System MTS 810 con una celda de carga de $100 \mathrm{kN}$ y a una velocidad de $10 \mathrm{~mm} / \mathrm{min}$. Las probetas de ensayo se prepararon en moldes de teflón en forma de cilindros con las dimensiones normalizadas por la ISO $5833\left(6 \mathrm{~mm}\right.$ de diámetro $\times 12 \mathrm{~mm}$ de altura). ${ }^{[6]}$ Las determinaciones se realizaron por quintuplicado. Para calcular la resistencia a la compresión (1) y el módulo de elasticidad (2) se utilizaron las Ecuaciones 1 y 2:

$$
\sigma[\mathrm{MPa}]=\frac{4 \mathrm{~F}}{\Pi \mathrm{d}^{2}} \times 10^{-6}
$$

Tabla 1. Rango de valores de los parámetros tiempo de fraguado y temperatura máxima de polimerización según lo establecido en la Norma ISO 5833.

\begin{tabular}{cc}
\hline $\begin{array}{c}\text { Tiempo de fraguado } \\
(\text { minutos })\end{array}$ & $\begin{array}{c}\text { Temperatura máxima de } \\
\text { polimerización }\left({ }^{\circ} \mathbf{C}\right)\end{array}$ \\
\hline $\mathrm{t}_{\mathrm{f}} \pm \Delta \mathrm{t}_{\mathrm{f}}$ & $\mathrm{T}_{\max } \pm \Delta \mathrm{T}_{\max }$ \\
$3-15$ & $90 \pm 5$ \\
\hline
\end{tabular}

Tabla 2. Diseño experimental aplicado.

\begin{tabular}{ccc}
\hline Nivel & A & B \\
\hline- & 10 & $1,5: 1$ \\
$1 / 2$ & 50 & $2: 1$ \\
+ & 90 & $2,5: 1$ \\
\hline C1 & Experimentos & $1 / 2$ \\
C2 & $1 / 2$ & + \\
C3 & + & $1 / 2$ \\
C4 & + & - \\
C5 & $1 / 2$ & - \\
C6 & + & + \\
C7 & $1 / 2$ & + \\
C8 & - & $1 / 2$ \\
C9 & - & - \\
\hline
\end{tabular}




$$
\mathrm{E}[\mathrm{GPa}]=\frac{\sigma}{\varepsilon}=\frac{\mathrm{FL}_{0}}{\Pi \mathrm{d}^{2} \Delta \mathrm{L}} \times 10^{-9}
$$

Donde F: es la carga máxima de rotura, expresada en Newton [N], d: el diámetro de la probeta, expresado en metros [m], $\mathrm{L}_{0}$ : longitud inicial de la probeta, expresada en metros $[\mathrm{m}], \Delta \mathrm{L}$ : cambio en la longitud de la probeta luego de ser sometida la carga $\left(\Delta \mathrm{L}=\mathrm{L}_{0}-\mathrm{L}_{\mathrm{f}}\right)$, expresada en metros $[\mathrm{m}]$.

\section{Caracterización termo analítica}

Se utilizó un calorímetro METTLER TC10A con unidad de DSC20 para la determinación de los parámetros térmicos del proceso de fraguado. Aproximadamente $30 \mathrm{mg}$ de cada muestra fueron colocados en cápsulas de aluminio herméticas. Se aplicó un régimen dinámico de calentamiento con una velocidad de $5{ }^{\circ} \mathrm{C} / \mathrm{min}$. Las determinaciones se realizaron por triplicado a una temperatura ambiente de $(23 \pm 1){ }^{\circ} \mathrm{C}$ y a una humedad relativa de $(48 \pm 1) \%$.

La temperatura fue medida cada 10 segundos con el objetivo de obtener un gráfico continuo como el que se muestra en la Figura 1. El tiempo de fraguado fue calculado como el tiempo en el que la temperatura alcanza el valor de $\mathrm{T}_{\mathrm{f}}$, acorde a la Ecuación 3.

$$
\mathrm{T}_{\mathrm{f}}=\frac{\mathrm{T}_{\max }+\mathrm{T}_{\mathrm{amb}}}{2}
$$

Donde: $\mathrm{T}_{\max }$ : es la temperatura máxima alcanzada $\left[{ }^{\circ} \mathrm{C}\right] \mathrm{y}$ $\mathrm{T}_{\text {amb }}$ : es la temperatura ambiente del local donde se realizan las determinaciones $\left[{ }^{\circ} \mathrm{C}\right]$.

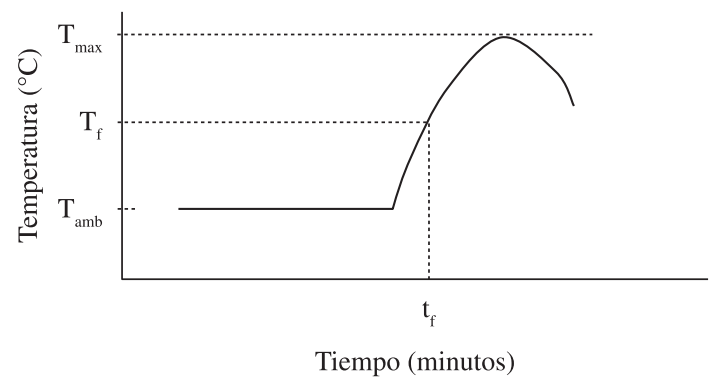

Figura 1. Curva típica de temperatura vs tiempo de fraguado.

\section{Bioactividad in vitro}

Para estudiar la interacción del material con el medio fisiológico se sumergieron 3 réplicas de dos formulaciones en fluido biológico simulado (FBS) durante 30 días con extracciones a los 15 días y al final del estudio. El FBS fue preparado según la formulación planteada por Kokubo y col. y posee una composición iónica similar a la del plasma sanguíneo humano. ${ }^{[11]}$ La relación volumen de FBS/área del cemento empleada en el estudio, fue de $10 \mathrm{~mL} / \mathrm{cm}^{2}$. El FBS se preparó disolviendo en agua bidestilada los nueve reactivos según el orden planteado en la Tabla 3 con agitación constante y teniendo en cuenta que cada reactivo debe añadirse después de la completa disolución del anterior.

Se empleó un Microscopio Electrónico de Barrido (MEB) Tescan TS 5130 SB con imágenes de electrones secundarios para observar la formación del crecimiento de capas apatíticas en la superficie, posterior a la inmersión en fluidos biológicos simulados. El análisis elemental cualitativo de los posibles crecimientos apatíticos se realizó mediante un equipo de Energía Dispersiva de Rayo X, EDX INCA 350 acoplado al MEB, con un potencial de trabajo de $20 \mathrm{kV}$.

Las muestras fueron montadas en porta muestras de aluminio y pegadas con discos de carbono, posteriormente se recubrieron con una película de oro/paladio aproximadamente dos minutos mediante la técnica de espolvoreado catódico.

\section{Resultados y Discusión}

\section{Caracterización mecánica}

El primer requerimiento que deben cumplir los cementos óseos acrílicos es una elevada resistencia a la compresión, lo cual se fundamenta en que es la principal solicitación que afecta al tejido óseo al realizar ejercicios tales como caminar o correr. Las propiedades mecánicas de las formulaciones en estudio se evaluaron a partir de la determinación de la Resistencia a la compresión axial y el Módulo de Young.

\section{Resistencia a la compresión axial}

Los valores de resistencia a la compresión axial $(\sigma)$ fueron calculados a partir de las curvas de fuerza/deformación obtenidas. Los tres composites con un $10 \%$ de $\mathrm{AcV}$ en su

\begin{tabular}{|c|c|c|c|c|}
\hline Ión & $\begin{array}{c}\text { Composición } \\
\text { FBS } \\
\end{array}$ & $\begin{array}{c}\text { Composición } \\
\text { Plasma sanguíneo }\end{array}$ & Reactivos & $\begin{array}{c}\text { Cantidad } \\
\text { (g) }\end{array}$ \\
\hline Sodio, $\mathrm{Na}^{+}$ & 142 & 142 & $\mathrm{NaCl}$ & 7,9960 \\
\hline Potasio, $\mathrm{K}^{+}$ & 5,0 & 5,0 & $\mathrm{NaHCO}_{3}$ & 0,3500 \\
\hline Magnesio, $\mathrm{Mg}^{2+}$ & 1,5 & 1,5 & $\mathrm{KCl}$ & 0,2240 \\
\hline Calcio, $\mathrm{Ca}^{2+}$ & 2,5 & 2,5 & $\mathrm{~K}_{2} \mathrm{HPO}_{4} \cdot 3 \mathrm{H}_{2} \mathrm{O}$ & 0,2280 \\
\hline Cloruro, $\mathrm{Cl}^{-}$ & 147,8 & 103,0 & $\mathrm{MgCl}_{2} \cdot 6 \mathrm{H}_{2} \mathrm{O}$ & 0,3050 \\
\hline Hidrógenocarbonato, $\mathrm{HCO}_{3}^{-}$ & 4,2 & 27 & $\mathrm{HCl} 1 \mathrm{~mol} / \mathrm{L}$ & $40 \mathrm{~mL}$ \\
\hline Hidrógenofosfato, $\mathrm{HPO}_{4}^{2-}$ & 1,0 & 1,0 & $\mathrm{CaCl}_{2}$ & 0,2780 \\
\hline \multirow[t]{2}{*}{ Sulfato, $\mathrm{SO}_{4}^{2-}$} & 0,5 & 0,5 & $\mathrm{Na}_{2} \mathrm{SO}_{4}$ & 0,0740 \\
\hline & & & $\mathrm{NH}_{2} \mathrm{C}\left(\mathrm{CH}_{2} \mathrm{OH}\right)_{3}$ & 6,0570 \\
\hline
\end{tabular}

Tabla 3. Composición del FBS y del plasma sanguíneo. Reactivos y cantidades a añadir. 
composición (Figura 2) presentaron propiedades superiores a las exigidas por la Norma ISO 5833 ( $\geq 70 \mathrm{MPa})$, lo que constituye un aspecto significativo para el desarrollo futuro de éstas formulaciones.

$\mathrm{Al}$ evaluar el efecto de la variable A: contenido de $\mathrm{AcV}$, se observa que sólo las formulaciones que presentan un $10 \%$ de dicho monómero cumplen con el mínimo que exige la Norma ISO 5833 (70 MPa) para cementos óseos acrílicos y a medida que en las formulaciones aumenta la incorporación de $\mathrm{AcV}$, la resistencia a la compresión axial disminuye. Este comportamiento puede explicarse debido a que existe sólo un $10 \%$ de $\mathrm{AcV}$ en estas formulaciones y conociendo la escasa reactividad de este monómero frente al MMA, indica que el cemento resultante estará muy enriquecido de poli (metacrilato de metilo), el cual es un polímero de cadena larga, hidrófobo, rígido y con un valor elevado de resistencia a la compresión. ${ }^{[12,13]}$

Por otra parte, es notable que la incorporación de AcV en porcientos iguales y superiores a 50, disminuye al doble los valores de resistencia a la compresión con respecto a C7, C8 y C9 (Figura 2). Dicho comportamiento puede estar asociado a la menor formación de PMMA a medida que aumenta el contenido de $\mathrm{AcV}$ en los cementos, dado

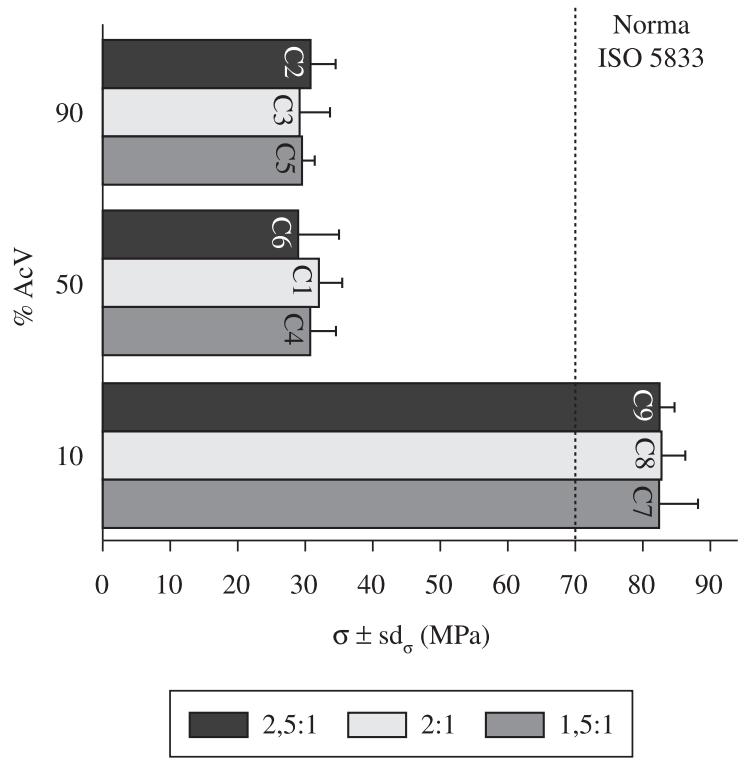

Figura 2. Resultados de la resistencia a compresión axial de las 9 formulaciones en estudio. que el $\mathrm{AcV}$, es un monómero más hidrofílico, tiende a disminuir considerablemente las propiedades mecánicas de los compuestos en cuestión. Una vez consumido todo el MMA presente, el radical de AcV formado en la reacción de iniciación, comienza a homopolimerizar, lo que trae consigo una notable disminución de la resistencia a la compresión.

$\mathrm{Si}$ analizamos la influencia que ejerce la relación $\mathrm{S} / \mathrm{L}$ sobre la variable en estudio se puede apreciar que la misma, no sufre variaciones apreciables cuando se emplea una $\mathrm{u}$ otra relación. Lo cual puede estar asociado a que las razones numéricas empleadas no difieren mucho entre sí.

Los resultados estadísticos avalan los obtenidos experimentalmente, pues la variable $\mathrm{B}$ : relación $\mathrm{S} / \mathrm{L}$ no influye de manera significativa sobre el modelo escogido (Tabla 4). El análisis de varianza indicó que la variable con más influencia sobre la resistencia a la compresión axial es el contenido de $\mathrm{AcV}$ presente en los composites, para un valor de $p<0,05$ y un coeficiente de determinación $\left(\mathrm{R}^{2}\right)$ de $98 \%$.

\section{Módulo de Young ó de elasticidad}

El módulo de elasticidad (E) se puede interpretar como la resistencia de los materiales a ser deformados cuando se le aplica una fuerza determinada ${ }^{[5]}$, por tanto, cuanto mayor es su valor, más resistencia a la deformación tienen los materiales ensayados. Los cementos evaluados permitieron obtener valores de módulo de elasticidad que oscilaron entre 0,4 y 2,1 GPa. Se observa en la Figura 3, la dependencia de la propiedad, con la incorporación de $\mathrm{AcV}$, es decir, a medida que esta aumenta disminuye el módulo de elasticidad.

Las formulaciones con mayores valores de Módulo de Elasticidad, son C7, C8 y C9, que en analogía con la propiedad mecánica discutida anteriormente, son las que presentan solamente un $10 \%$ de $\mathrm{AcV}$ en la composición del cemento, por lo que aquellas formulaciones con mayores valores de resistencia a la compresión son las que poseen niveles de módulos de elasticidad superiores, lo cual presupone una mayor rigidez.

Nótese la tendencia a disminuir el módulo de elasticidad a medida que aumenta en los cementos el contenido de $\mathrm{AcV}$, así como una estabilización de esta propiedad en aquellas formulaciones con cantidades de $\mathrm{AcV}$ superiores al $50 \%$. Dicho comportamiento se explica por los argumentos expuestos en el análisis de los resultados de resistencia a la compresión, ya que cuanto mayor es el módulo de elasticidad,

Tabla 4. Análisis de varianza (ANOVA) del ensayo de resistencia a la compresión.

\begin{tabular}{|c|c|c|c|c|c|}
\hline \multicolumn{6}{|c|}{ Respuesta: Resistencia a la compresión } \\
\hline \multicolumn{6}{|c|}{ Tabla de ANOVA para el Modelo Factorial Seleccionado } \\
\hline \multicolumn{6}{|c|}{ Tabla de análisis de varianza [Suma de cuadrados parciales] } \\
\hline Fuente & Suma de Cuadrados & GL & Cuadrado Medio & Valor de F & Prob > F \\
\hline Modelo & 7308.58 & 6 & 1218.10 & 91.53 & $<0.0001$ significativo \\
\hline A & 7262.67 & 4 & 1815.67 & 136.43 & $<0.0001$ \\
\hline \multirow[t]{2}{*}{ Residual } & 106.46 & 8 & 13.31 & & \\
\hline & & & R-Cuadrado & 0.9856 & \\
\hline
\end{tabular}


más resistencia a la deformación ofrecen los materiales ensayados, dependiendo de esto la rigidez del material.

Las relaciones $\mathrm{S} / \mathrm{L}$ empleadas en este ensayo no aportaron variaciones apreciables en los valores obtenidos de Módulo de Young, lo cual puede ser explicado por las mimas razones expuestas anteriormente.

El análisis de varianza (Tabla 5) ratificó que sólo la variable (A) ejerce una influencia significativa sobre el Módulo de Elasticidad, debido a que presenta un valor de $p$ menor que 0,05. Por otro lado, se evidenció que estadísticamente las relaciones $\mathrm{S} / \mathrm{L}$ empleada en cada formulación no ejercen influencia significativa, ya que dicha variable presentó valores de $p$ superiores al $\alpha$ elegido, para un coeficiente de determinación $\left(\mathrm{R}^{2}\right)$ de $98 \%$.

El efecto de las interacciones entre las variables de estudio (Figura 4), para ambas pruebas mecánicas reporta una línea recta en el intervalo entre 10 y $50 \%$ con dependencia exclusiva del contenido de AcV. Lo cual, quiere decir que hasta un $50 \%$, a medida que aumente el porciento de $\mathrm{AcV}$ en las formulaciones, los valores de $\sigma$ y $\mathrm{E}$ se incrementaran linealmente. Es importante destacar que tres de los composites
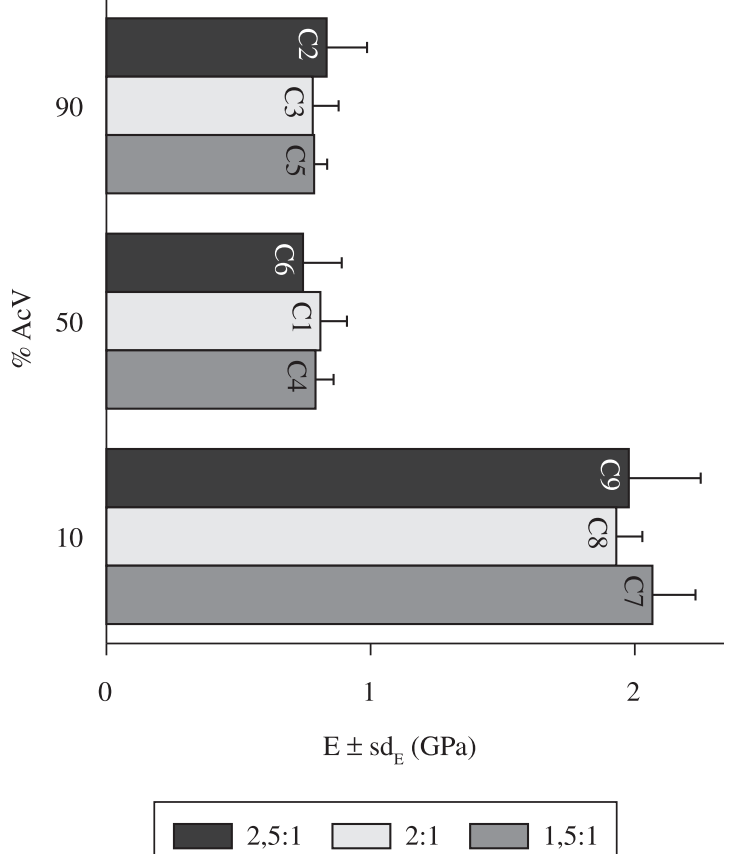

Figura 3. Resultados del módulo de Young de las formulaciones en estudio. desarrollados cumplen con lo establecido por la Norma ISO 5833 en cuanto a resistencia a la compresión axial, los cuales además, poseen valores adecuados de módulo de elasticidad, lo que pudiera validar su uso futuro como cementos óseos acrílicos.

\section{Caracterización termoanalítica}

El fraguado de los cementos óseos acrílicos es un proceso complejo porque el contacto entre la parte sólida con la líquida marca el inicio de eventos químicos y físicos que tienen lugar simultáneamente. La solvatación de las perlas poliméricas por la disolución monomérica y la difusión del líquido en la fase sólida son algunos de los eventos físicos que ocurren. El más importante proceso químico que tiene lugar es la polimerización del MMA. Cuando la reacción de polimerización comienza, el monómero de MMA es consumido rápidamente contribuyendo a acelerar la reacción de polimerización exotérmica y por lo tanto alcanza más rápidamente el "efecto gel". ${ }^{[14]}$

\section{Tiempo de fraguado}

El tiempo de fraguado es el tiempo en el cual la mezcla polimérica puede ser manipulada y aplicada en el sitio de implantación sin problemas. En nuestro estudio, se obtuvieron composites con tiempos de curado o fraguado $\left(\mathrm{t}_{\mathrm{f}}\right)$ en un rango entre 3,47 y 6,34 minutos, acorde a lo establecido para su aplicación como cementos quirúrgicos en fijaciones de prótesis y sustituciones óseas ${ }^{[6]}$ (Figura 5).

Para una misma relación $\mathrm{S} / \mathrm{L}$, los mayores tiempos de fraguado se obtienen en las formulaciones que contiene un $90 \%$ de AcV. Esto se debe a que dichos cementos están menos enriquecidos de MMA y por tanto la reacción de fraguado del cemento óseo (donde el MMA es el reaccionante fundamental) se dificulta por la presencia de apreciables cantidades de AcV. De ahí que a medida que exista más contenido de $\mathrm{AcV}$ en las formulaciones óseas acrílicas sea necesario más tiempo para concluir la reacción de fraguado. Tanto es así que las formulaciones que mostraron los menores valores de $t_{f}$, se encuentran relacionadas con las que presentan en su composición un $90 \%$ de MMA y solamente un $10 \%$ de AcV.

Se aprecia además, que no se obtuvo ninguna dependencia en los valores de los tiempos de fraguado con la relación $\mathrm{S} / \mathrm{L}$ empleada. Igualmente, el rango obtenido entre 3,47 y 6,34 minutos es muy pequeño, lo cual quiere decir que las

Tabla 5. Análisis de varianza (ANOVA) del ensayo de módulo de Young.

\begin{tabular}{|c|c|c|c|c|c|}
\hline \multicolumn{6}{|c|}{ Respuesta: Módulo de Young } \\
\hline \multicolumn{6}{|c|}{ Tabla de ANOVA para el Modelo Factorial Seleccionado } \\
\hline \multicolumn{6}{|c|}{ Tabla de Análisis de varianza [Suma de cuadrados parciales] } \\
\hline Model & 3.83 & 6 & 0.64 & 75.66 & $<0.0001$ significativo \\
\hline A & 3.82 & 4 & 0.96 & 113.17 & $<0.0001$ \\
\hline \multirow[t]{2}{*}{ Residual } & 0.068 & 8 & $8.445 \mathrm{E}-003$ & & \\
\hline & & & R-Cuadrado & 0.9827 & \\
\hline
\end{tabular}




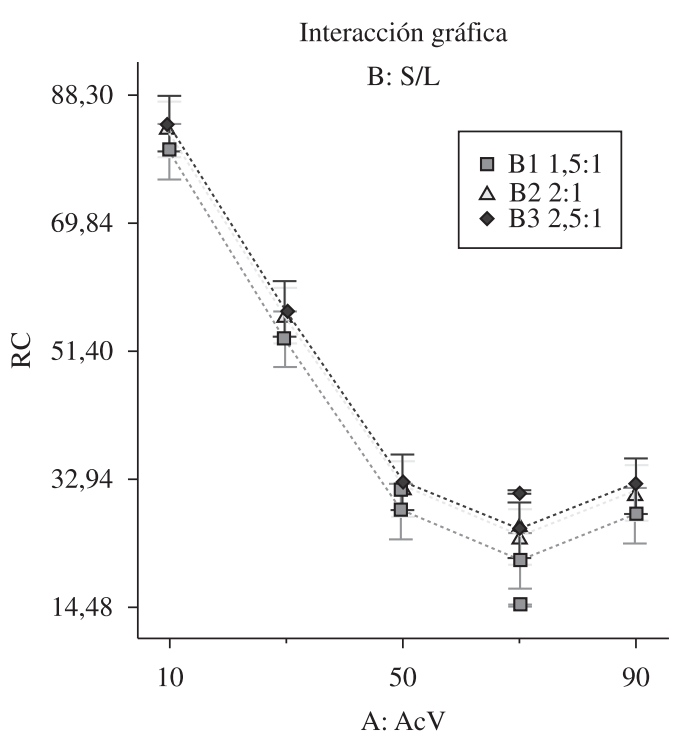

(a)

Figura 4. Gráfico de las interacciones de las variables en estudio.

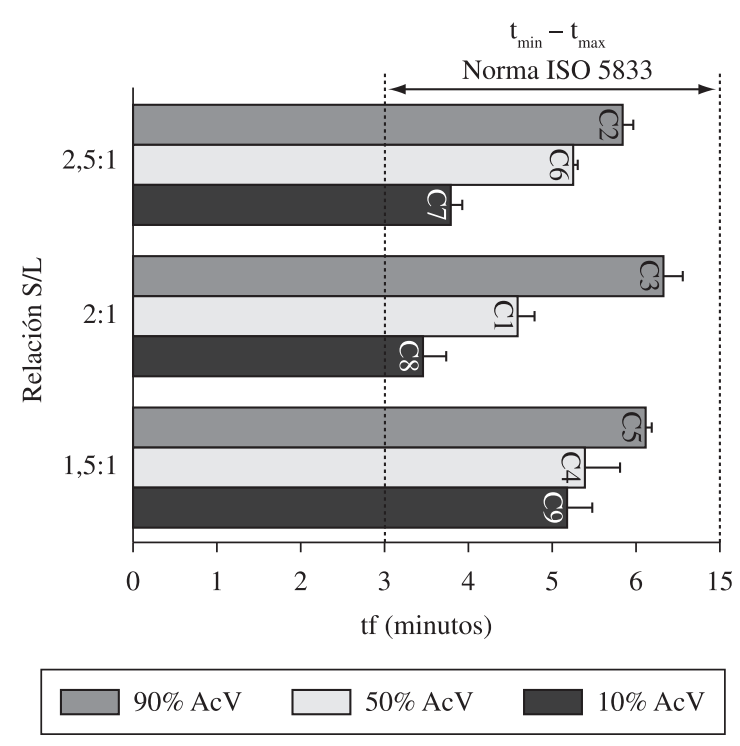

Figura 5. Tiempo de fraguado de las formulaciones en estudio.

variaciones impuestas a esta variable no son lo suficientemente amplias como para provocar resultados apreciablemente diferentes.

Los resultados estadísticos corroboran lo obtenido experimentalmente, ya que el análisis de varianza (Tabla 6), evidenció que solamente la variable A (contenido de AcV), ejerce una influencia significativa sobre el tiempo de fraguado, presentando un valor de $p$ menor de 0,05 para un coeficiente de determinación $\left(\mathrm{R}^{2}\right)$ de $95 \%$.

\section{Temperatura máxima de polimerización}

La temperatura máxima de polimerización $\left(\mathrm{T}_{\max }\right)$ es un parámetro muy importante a tener en cuenta para evitar la necrosis tisular de los tejidos adyacentes al implante. Es por esto que las normas internacionales han establecido el valor

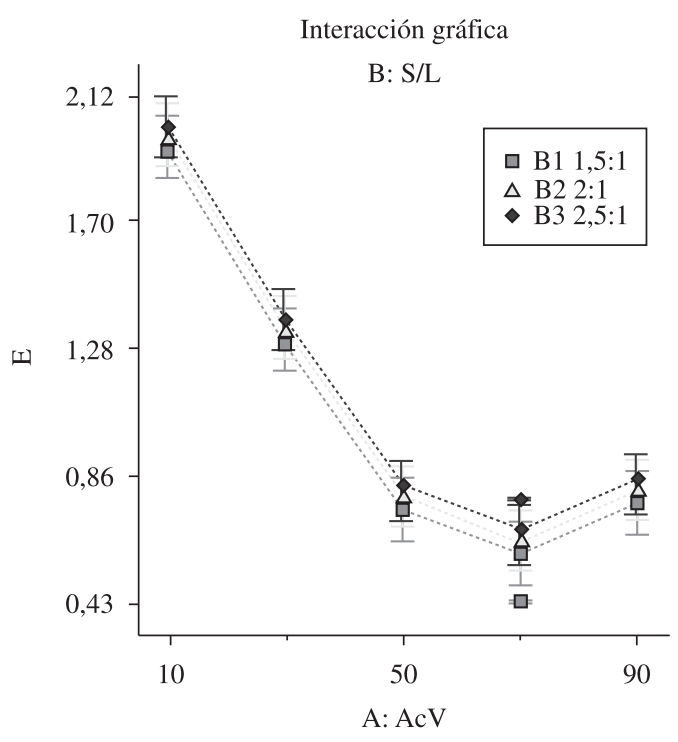

(b)

de $(90 \pm 5){ }^{\circ} \mathrm{C}$ como máximo para este parámetro, con el objetivo de que los investigadores tengan un control sobre los ensayos de los materiales en estudio.

Como puede apreciarse (Figura 6), todas las formulaciones en estudio, satisfacen las exigencias de la Norma ISO 5833. ${ }^{[6]}$ Se obtuvieron composites con temperaturas máximas de polimerización en un rango entre 60 y $88^{\circ} \mathrm{C}$ aproximadamente.

$\mathrm{Si}$ analizamos la influencia de la variable A: contenido de $\mathrm{AcV}$, sobre la temperatura máxima de polimerización observamos que los cementos con mayores contenidos de $\mathrm{AcV}$ presentan los mayores valores de $\mathrm{T}_{\max }$ al igual que en el tiempo de fraguado. Este comportamiento puede deberse a la presencia de un segundo monómero, que provocaría un aumento considerable en la temperatura máxima del proceso de fraguado de los cementos óseos, debido a la variación en la velocidad de polimerización. Tanto un aumento acelerado como gradual de la $\mathrm{T}_{\max }$ de polimerización no son recomendados una vez implantados estos materiales, ya que como se ha mencionado puede provocar necrosis del tejido adyacente.

Una vez más las relaciones S/L empleadas no tuvieron influencia significativa sobre la variable en estudio (Tabla 7) presentando un valor de $p$ mayor de 0,05 . No obstante, existen autores ${ }^{[15]}$ que plantean que un mayor volumen de fase líquida en la preparación de los cementos da como resultado mayores valores de $\mathrm{T}_{\max }$, ya que esto posibilita un mejor proceso de mezclado de ambas fases, contribuyendo a acelerar la reacción de polimerización exotérmica y por tanto se alcanza más rápidamente el "efecto gel". En nuestro estudio, la parte líquida se mantuvo constante, sin embargo, las formulaciones preparadas a partir de la relación S/L 1,5:1 son las que, comparadas con las otras relaciones empleadas, presentan mayor parte líquida; y en similitud con lo anteriormente discutido mostraron, los mayores valores de $\mathrm{T}_{\max }$, resultado que concuerda con lo anteriormente planteado. 
Tabla 6. Análisis de varianza (ANOVA) del ensayo de tiempo de fraguado.

\begin{tabular}{cccccc}
\hline \multicolumn{7}{c}{ Respuesta: Tiempo de fraguado } \\
\hline \multicolumn{7}{c}{ Tabla de ANOVA para el Modelo Factorial Seleccionado } \\
\hline \multicolumn{7}{c}{ Tabla de Análisis de varianza } & [Suma parcial de cuadrdos] \\
\hline Fuente & Suma de Cuadrados & GL & Cuadrado Medio & Valor F & Prob > F \\
\hline Model & 7.90 & 4 & 0.99 & 7.93 & 0.0207 significativo \\
A & 5.73 & 2 & 2.86 & 9.73 & 0.0291 \\
B & 0.99 & 2 & 0.50 & 1.68 & 0.2948 \\
Residual & 2.17 & 4 & 0.36 & 0.9524 \\
\end{tabular}

Tabla 7. Análisis de varianza (ANOVA) del ensayo de temperatura máxima de polimerización.

\begin{tabular}{|c|c|c|c|c|c|}
\hline \multicolumn{6}{|c|}{ Respuesta: Temperatura máxima de polimerización } \\
\hline \multicolumn{6}{|c|}{ Tabla de ANOVA para el Modelo Factorial Seleccionado } \\
\hline \multicolumn{6}{|c|}{ Tabla de Análisis de varianza [Suma de cuadrados parciales] } \\
\hline Fuente & Suma de Cuadrados & GL & Cuadrado Medio & Valor F & Prob $>$ F \\
\hline Model & 670.78 & 4 & 167.70 & 5.44 & 0.0367 significativo \\
\hline A & 530.35 & 2 & 265.17 & 8.60 & 0.0346 \\
\hline $\mathrm{B}$ & 140.44 & 2 & 140.40 & 2.28 & 0.2187 \\
\hline \multirow[t]{2}{*}{ Residual } & 123.36 & 4 & 30.84 & & \\
\hline & & & R-Cuadrado & 0.9447 & \\
\hline
\end{tabular}

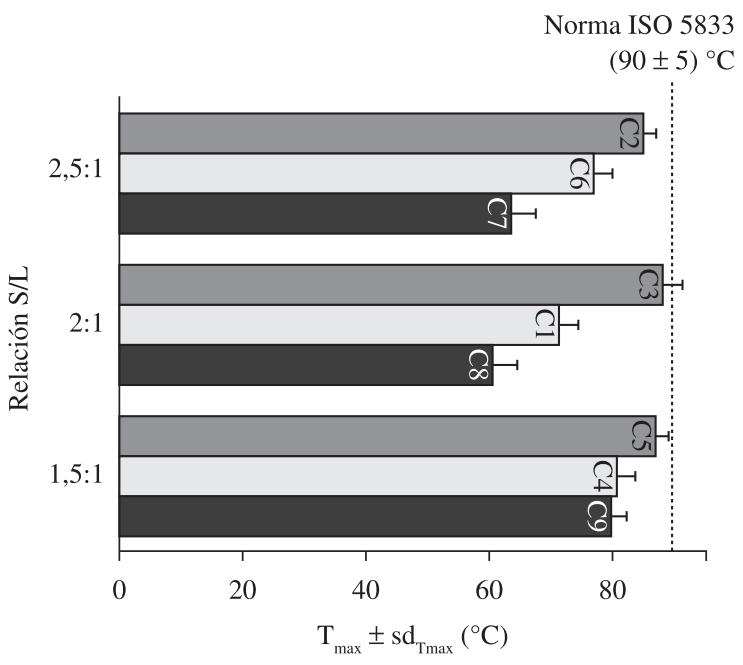

$90 \% \mathrm{AcV}$

$50 \% \mathrm{AcV}$

$10 \% \mathrm{AcV}$

Figura 6. Temperaturas máximas de polimerización de las formulaciones en estudio.

De manera general, los valores de temperatura máxima de polimerización presentaron un comportamiento similar al tiempo de fraguado y se observa por el valor medio obtenido $\left(77^{\circ} \mathrm{C}\right)$ la poca dispersión de los valores de las temperaturas obtenidas.

\section{Estudios de bioactividad en superficies}

La formación de una capa apatítica en la superficie de un material indica que el mismo podría presentar un comportamiento bioactivo por la existencia de núcleos de
$\mathrm{Ca}^{2+}$ pertenecientes a la hidroxiapatita, lo cual redunda en una mejor adhesión del nuevo tejido al material y por tanto en una mayor eficiencia del implante. ${ }^{[16]}$

El cemento óseo acrílico es un biomaterial no bioactivo, su matriz polimérica es altamente hidrofóbica por lo que debilita el contacto carga-solución y dificulta el crecimiento del nuevo tejido. La Figura 7 a y b muestran las micrografías de la superficie de una muestra acrílica sin acetato de vinilo (solamente PMMA + 30\% de HAP-200), usada como control en este estudio, a los 15 y 30 días de inmersión en solución de fluido biológico simulado (FBS). A los 15 días (Figura 7a) se aprecia claramente la interconexión entre las partículas de PMMA que muestran su forma esférica así como la presencia de algunas partículas cristalinas (zonas claras). Ya a los 30 días (Figura 7b) se manifiesta ligera bioactividad dada por la presencia de ciertos crecimientos apatíticos en la superficie de la muestra.

Se decidió estudiar la bioactividad in vitro de las formulaciones $\mathrm{C} 3$ y C8, con 90 y $10 \%$ de $\mathrm{AcV}$ respectivamente y una relación $\mathrm{S} / \mathrm{L}$ de 2:1.

En la Figura 8a-c se puede observar la superficie de la formulación C3 (90\% AcV) en diferentes tiempos de inmersión en la solución de fluido biológico simulado (FBS). A los 15 días del estudio existe una pérdida de fronteras entre las partículas poliméricas y los rellenos cerámicos. Se observan zonas oscuras circulares rodeadas de paredes menos oscuras y más texturadas donde se aprecia nítidamente partículas más claras que pudieran ser productos formados preferencialmente sobre estas zonas.

Al cabo de los 30 días dejan de observarse las perlas de PMMA, cubriéndose toda la superficie por "colonias" de 


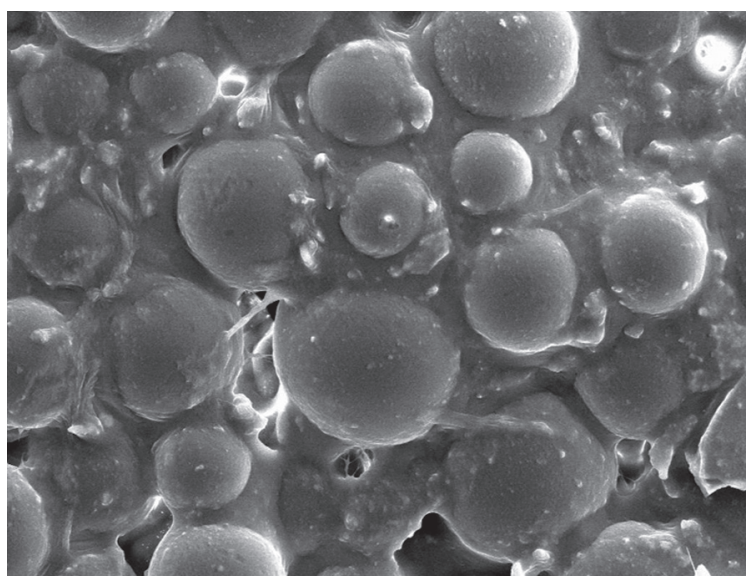

15 días en SBF (1000x)

(a)

Figura 7. Micrografías de la muestra usada como control.

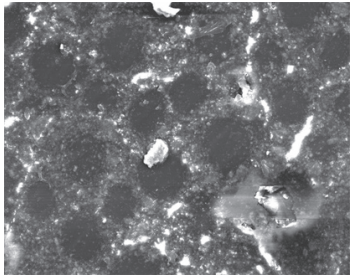

C3: 15 días en SBF (1000x)

(a)

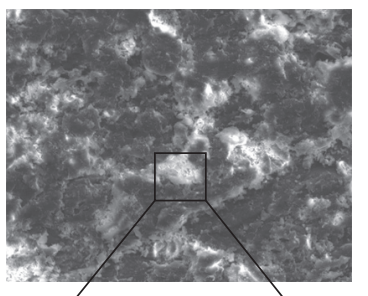
C3:30 días en SBF (1000x)

(b)

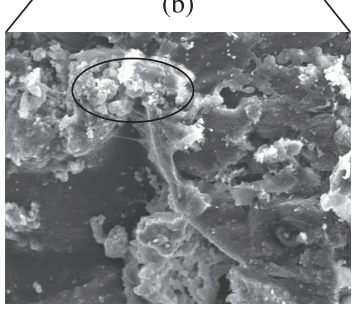

C3: 30 días en $\mathrm{SBF}(5000 \times)$

(c)

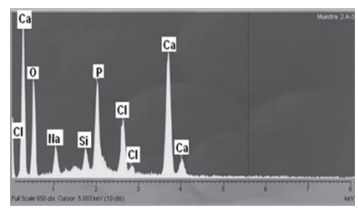

(d)

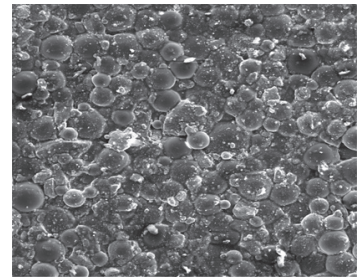

C8: 15 días en SBF (1000x)

(e)

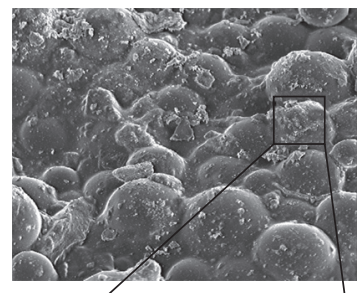

C8: 30 días en SBF (1000x)

(f)

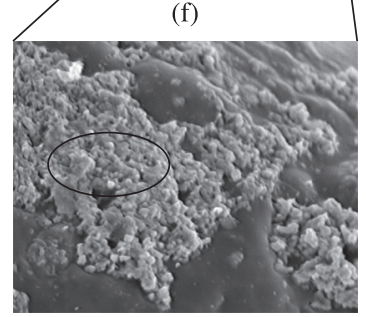

C8: 30 días en SBF (5000×)

(g)

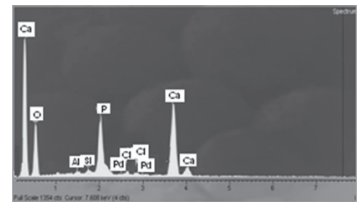

(h)
Figura 8. Micrografías de la muestra C3 (a-c) y C8 (e-g). Análisis de la composición elemental de la zona señalada $(\mathrm{d}, \mathrm{h})$.

estructuras apatíticas. En una imagen ampliada a 5000x (Figura 8c) se pueden observar estas estructuras en forma de

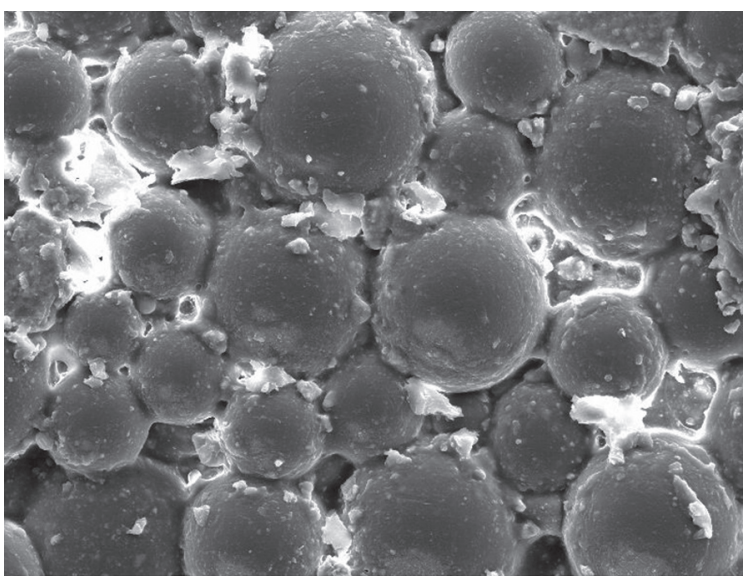

30 días en SBF $(1000 \times)$

(b)

rosetas, las cuales son análogas a las reportadas en materiales con matrices poliméricas hidrofílicas con cargas similares. ${ }^{[17,18]}$

El análisis elemental de una de estas estructuras pudo comprobar que la misma está constituida fundamentalmente por la presencia de calcio y fósforo, típico de las estructuras apatíticas, lo que corrobora el carácter bioactivo de esta formulación. Los picos restantes que aparecen en el espectro EDX (Figura 8d) corresponden a otros componentes de la disolución de FBS.

La formulación C3 contiene un elevado contenido de monómero hidrofílico (AcV) lo que aumenta la posibilidad de contacto de los núcleos o semillas formados con los fluidos, así como el posterior crecimiento y coalescencia de estos cristales. El aumento de la biocompatibilidad por esta vía se hace a veces a expensas de sacrificar las propiedades mecánicas del cemento, las cuales son también muy importantes dada la aplicación a que están destinados estos materiales.

En la Figura 8e-g se aprecia la morfología de la formulación C8 (10\% de AcV), uno de los cementos que presenta las mejores propiedades mecánicas. A los 15 días de inmersión en FBS, no se aprecian grandes cambios morfológicos con respecto a la muestra control (Figura 7a). Ya a los 30 días se observa un crecimiento selectivo de cristales rodeando a las perlas de PMMA, lo que pudiera indicar que dicha formulación en un período de tiempo mayor podría también presentar un futuro comportamiento bioactivo.

El análisis de la composición elemental de de uno de estos crecimientos (Figura 8g) después de 30 días de inmersión en FBS, evidenció la presencia de calcio y fósforo, pero en menor grado que en la formulación anterior ya que en los espectros de EDX (Figura 8h) los picos relacionados con dichos iones son menos intensos.

La influencia de la incorporación de monómero hidrofílico en cementos óseos acrílicos las sobre el comportamiento bioactivo puede notarse al comparar las micrografías de la formulación que contiene $90 \%$ de acetato de vinilo (Figura 8a-c) con las micrografías de la muestra control 
(Figura 7a-b). La ausencia de una capa apatítica en la superficie de la muestra que no contiene $\mathrm{AcV}$ al trascurrir 30 días de ensayos, indica que la presencia de acetato de vinilo en los cementos óseos acrílicos, aporta un carácter hidrofílico a los mismos, lo que trae consigo un mejor contacto disoluciónbiomaterial y por ende una mayor deposición de iones $\mathrm{Ca}^{+2} \mathrm{y}$ $\mathrm{PO}_{4}^{-3}$ en la superficie de los cementos.

\section{Conclusiones}

Las formulaciones con un $10 \%$ de incorporación del monómero $\mathrm{AcV}$ (C7, C8 y C9) presentaron los mayores valores de resistencia a la compresión axial. Dichas formulaciones también poseen los mayores niveles de módulo de elasticidad, lo cual presupone una mayor rígidez. Todos los composites ensayados cumplieron con las exigencias de curado descritas en la Norma ISO 5833, pero presentan un menor tiempo de fraguado las que contienen un $10 \%$ de $\mathrm{AcV}$, coincidentemente las de menor temperatura máxima de polimerización. Estadísticamente de las dos variables en estudio, sólo ejerció una influencia significativa en todos los ensayos, el contenido de acetato de vinilo. Se demostró de manera preliminar por inmersión de las muestras en FBS, que la inclusión de acetato de vinilo en cementos óseos acrílicos favorece la formación de capas apatíticas ricas en calcio y fósforo en la superficie del material.

\section{Referencias Bibliográficas}

1.López, M. - Rev. CENIC Cienc. Quim., 37, p. 77 (2006).

2. Morejón, L.; Delgado, J. A.; Mendizábal, E.; Davivenko, N.; Quiroz, A.; Pau, M. \& Gil, J. - Rev. CENIC Cienc. Quim., 37, p. 155 (2006).

3. Tunney, M.; Jones, D. \& Gorman, S. - Int. J. Pharm., 151, p. 121 (1997).

4. Virto, M.; Frutos, P.; Torrado, S. \& Frutos, G. - Biomaterials, 24, p. 79 (2003).

5. Cuadrado, T. R. \&Abraham, G. - "Propiedades mecánicas de Biomateriales", in: Biomateriales, cap. 8, Sastre, R.; Aza, S. \& San Román, J. (eds.), Faenza Editrice Ibérica, Italia (2004).
6. INTERNATIONAL STANDARD ORGANIZATION - ISO - "ISO 5833: implants for surgery-Acrylic resin cement", Switzerland (2002).

7. AMERICAN SOCIETY FOR TESTING AND MATERIALS STANDARDS - ASTM - "ASTM F541: standard specification for acrylic bone cement", West Conshohocken (1999).

8. Meyer, P.; Lautenschlager, E. \& Moore, B. - J. Bone Joint Surg., 55A, p. 149 (1973).

9. Morejón, L.; Mendizábal, E.; Delgado, J. \& Davidenko, N. - Lat. Am. App. Res., 35, p. 175 (2005).

10. González, R.; Melo, M.; Rodríguez, A. \& Pérez, A. - Quim. Nova, 16, p. 509 (1993).

11. Kim, H.; Miyazaki, T.; Kokubo, T. \& Nakamura, T. - Key Engineering Material, 47, p. 192 (2001).

12. Serbetci, K.; Korkusuz, F. \& Hasirci, N. - Polym.Test., 23, p. 145 (2004).

13. Shinzato, S.; Neo, M.; Kokubo, T. \& Nakamura, T. - J. Biomed. Mater. Res., 53, p. 51 (2000).

14. Lopez, M.; Fuentes, G.; González, R.; González, J.; Peón, E. \& Toledo, C. - Lat. Am. App. Res., 38, p. 228 (2008).

15. Shinzato, S.; Nakamura, T.; Tamura, J.; Kokubo, T. \& Kitamura, Y. - J. Biomed. Mater. Res., 56, p. 571 (2001).

16. Espigares, I.; Elvira, C.; Mano, J.; Vazquéz, B.; San-Román, J. \& Reis, R. - Biomaterials, 23, p. 1883 (2002).

17. Tsukeoka, T.; Suzuki, M.; Ohtsuki, Ch.; Supino, A.; Tsuneizumi, Y. \& Kuramoto, K. - Biomaterials, 27, p. 3897 (2006).

18. Miyazaki, T.; Ohtsuki, H.; Kyomoto, M.; Tanihara, M.; Mori, K. \& Kuramoto, K. - J. Biomed. Mater. Res. A, 67, p. 1417 (2003).

Enviado: 30/05/09

Reenviado: 22/07/09

Aceito: $04 / 01 / 10$

DOI: $10.1590 / \mathrm{S} 0104-14282010005000015$ 